\title{
Politique
}

Politique

\section{Les fonctions du député : bilan des réformes parlementaires à Québec}

\section{Réjean Pelletier}

Numéro 6, automne 1984

La réforme des institutions politiques

URI : https://id.erudit.org/iderudit/040464ar

DOI : https://doi.org/10.7202/040464ar

Aller au sommaire du numéro

Éditeur(s)

Société québécoise de science politique

ISSN

0711-608X (imprimé)

1918-6584 (numérique)

Découvrir la revue

Citer cet article

Pelletier, R. (1984). Les fonctions du député : bilan des réformes parlementaires à Québec. Politique, (6), 145-164. https://doi.org/10.7202/040464ar d'utilisation que vous pouvez consulter en ligne.

https://apropos.erudit.org/fr/usagers/politique-dutilisation/ 


\title{
Les fonctions du député: bilan des réformes parlementaires à Québec
}

\author{
Réjean PELLETIER \\ Université Laval
}

On a de plus en plus l'habitude d'attribuer au député québécois un triple rôle de législateur, de contrôleur et d'intermédiaire. ${ }^{1}$ Chacun de ces rôles doit être qualifié de diverses façons afin d'en comprendre la portée exacte. La perception que le député peut avoir de ces rôles et surtout l'exercice effectif de chacun d'entre eux sont influencés aussi bien par certains traits personnels du député que par la configuration socio-économique de sa circonscription ainsi que par son appartenance à la majorité parlementaire ou à l'opposition.

\section{Des rôles en mutation}

Si l'on s'attache d'abord au pouvoir d'initiative en matière de législation, il faut convenir que, dans notre système parle-

1. Voir Gaston Deschênes, Le député québérois, Québec, Assemblée nationale du Québec, 1979, 25-36. Dans une perspective un peu différente, André Bernard, La politique au Canada et au Québec, Montréal, P.U.Q., 1977, $501-510$ et André Gélinas, Les parlementaires et l'administration au Québec, Québec, P.U.L., 1969. Également Robert J. Jackson et Michael M. Atkinson, The Canadian Legislative System, Toronto, Macmillan of Canada, $2{ }^{\circ}$ édit., 1980; le numéro spécial du Journal of Canadian Studies / Revue d'études canadiennes, vol. 14, no 2, été 1979; William A.W. Neilson et James C. MacPherson, The Legislative Process in Canada. The Need for Reform, Montréal, Institut de recherches politiques, 1978. 
mentaire, ce pouvoir qui appartenait autrefois au monarque et à ses conseillers immédiats, a été transféré non pas au Parlement, mais au conseil des ministres secondé par la haute fonction publique. Certes, le Parlement est toujours souverain au sens où il constitue la seule instance habilitée à adopter la législation. Mais cette souveraineté se réduit à un rôle passif puisqu'il doit habituellement se contenter de réagir aux différents projets de loi que lui soumet le conseil des ministres. Devant la croissance des interventions de l'État dans nos sociétés modernes et face à la complexité accrue des problèmes, les gouvernements se sont entourés d'une fonction publique de plus en plus nombreuse et de plus en plus efficace. Ils deviennent ainsi mieux équipés et mieux informés que la plupart des députés sur des problèmes sans cesse plus complexes dont les solutions requièrent des analyses approfondies. C'est ainsi que le passage de l'État-gendarme à l'État-providence a sonné le glas d'une souveraineté déjà défaillante du Parlement. ${ }^{2}$

Le Québec n'a pas échappé à ces mutations. Le seul véritable législateur, c'est désormais le gouvernement qui définit pratiquement le programme législatif du Parlement appelé ensuite à discuter et à entériner les décisions prises à cet autre niveau. Ce rôle de législateur dévolu au gouvernement est renforcé et légitimé par la signification réelle d'une élection dans notre système parlementaire où l'électorat est appelé à choisir entre des partis qui sollicitent ses suffrages plutôt qu'entre des candidats en fonction de leurs qualités personnelles. Le gouvernement se considère alors

2. Voir André Chandernagor, Un Parlement, pour quoi faire?, Paris, Gallimard, Coll. Idées, 1967; Bernard Crick, The Reform of Parliament, Londres, Weidenfeld and Nicolson, 1964; Ronald Butt, The Power of Parliament, Londres, Constable, 1967. Pour le Canada, John Stewart, The Canadian House of Commons; Pracedure and Reform, Montréal, McGill-Queen's University Press, 1977 et surtout Thomas D'Aguino, G. Bruce Doern et Cassandra Blair, Le Gouvernement parlementaire au Canada; évaluation critique et propositions de changement, Ottawa, Interconsel L.td., 1979. 
à bon droit comme l'émanation du pays à la suite du choix effectué par l'électorat entre les différents partis politiques. ${ }^{3}$

On est ainsi passé d'un parlementarisme flexible lorsque la Chambre pouvait choisir son exécutif et le défaire éventuellement à un parlementarisme rigide ou structuré avec la montée des partis politiques disciplinés ${ }^{4}$. Désormais, c'est l'électorat qui choisit le parti appelé à former le gouvernement et c'est également l'électorat et non plus la Chambre qui pourra le défaire éventuellement. Cette règle s'impose tellement que, même dans les périodes de crise marquées par des gouvernements minoritaires, une défaite en Chambre ou l'anticipation d'une défaite conduit à un appel au peuple plutôt qu'à un changement de gouvernement. ${ }^{5}$ L'électorat et non la Chambre est alors appelé à trancher.

On peut ainsi dégager quelques traits fondamentaux de notre système parlementaire. La règle qui s'impose, c'est la domination de l'exécutif incarné à une époque par le monarque et ses conseillers et assimilé de nos jours aux différents gouvernements en place. L'exception, c'est la domination du législatif, soit au moment de la conquête du gouvernement responsable et dans les années qui suivirent avant l'apparition des partis politiques bien structurés et disciplinés.

C'est dans cette même perspective qu'il faut replacer le rôle du député comme contrôleur si l'on veut en saisir la portée exacte. Puisque le cabinet est devenu le véritable initiateur des politiques et qu'en retour, il doit en porter la responsabilité devant la po-

3. Ce thème est développé plus longuement dans Réjean Pellerier, «Le dépuré, un législateur défaillant?", dans Journal of Canadian Studies / Revue d"études canadiennes, vol. 14 , no 2 , été $1979,48-56$.

4. Voir Thomas A. Hockin, "Flexible and Structured Parliamentarism", Journal of Canadian Studies / Revue d'études canadiennes, vol. 14, no 2, été 1979, 8-17.

5. Les défaites en Chambre du gouvernement Diefenbaker en 1963 et du gouvernement Clark en 1979 ont entraîné la dissolution du Parlement et la convocation d'élections. C'est la population canadienne qui a été appelée à trancher entre les différents partis politiques. 
pulation, le contrôle des décisions et actions entreprises par le gouvernement s'exerce en dernier ressort par l'électorat à intervalles plus ou moins fréquents. Entre-temps, les parlementaires vont exercer leur rôle de contrôleurs du gouvernement et de l'administration d'une façon différente selon leur appartenance à l'équipe ministérielle ou au(x) parti(s) d'opposition. Comme l'a déjà montré André Gélinas, les membres de l'opposition sont de façon plus spécifique des contrôleurs de l'administration et de la politique ministérielle, alors que les députés du parti majoritaire sont plutôt des propagandistes à la fois de l'administration et de la politique ministérielle. ${ }^{6}$

C'est ainsi que les différents mécanismes de contrôle de l'action gouvernementale - comme les questions orales et écrites, l'examen des engagements financiers ou l'étude des crédits profitent surtout aux députés de l'opposition. Manifestement les députés du parti ministériel ne cherchent pas, sauf exceptions, à surveiller et critiquer les actions gouvernementales et le travail de l'administration. Une tendance se dessine cependant depuis quelques années visant à remettre en cause les interventions trop massives de l'État au sein de la société québécoise et la croissance trop prononcée de la machine administrative ainsi que sa lourdeur et sa trop grande complexité. De telles critiques se traduisent souvent de la part des députés par la recherche d'un contrôle effectif sur l'appareil administratif et sur les sociétés d'État.

À ce premier volet d'une nouvelle conception de la responsabilité gouvernementale selon laquelle le contrôle ultime est exercé par l'électorat plutôt que par la Chambre, s'ajoute un second volet relatif à l'efficacité du gouvernement. Celui-ci se montrera responsable lorsqu'il adoptera des politiques ou prendra des mesures qui seront adoptées sans grandes difficultés par le Parlement. Plus il dominera la Chambre, plus il se montrera

6. André Gélinas, op. cit., 101. 
efficace, plus il agira alors en gouvernement capable de prendre ses responsabilités, non pas de les fuir. C'est avant tout sur ses réalisations qu'il sera jugé ainsi que sur sa façon de gérer la société. Le taux de satisfaction qui en résultera au sein de la population va constituer le meilleur indice de la réélection ou de la défaite du parti au pouvoir.

Législateur défaillant et contrôleur encore mal assuré, le député s'est souvent réfugié dans son rôle d'intermédiaire ou dans ce que l'on pourrait appeler plus prosaïquement le «travail de comté». C'est un rôle qui pourra accaparer une bonne partie de son temps et de ses énergies, surtout dans les circonscriptions moins urbanisées. C'est un rôle qu'il remplira également avec plus d'enthousiasme s'il se perçoit surtout comme un mandataire chargé de transmettre les désirs et les opinions de ses électeurs ${ }^{7}$ plutôt qu'un fiduciaire cherchant à faire valoir ses idées personnelles sur des problèmes d'intérêt public.

Ce rôle d'intermédiaire comporte de multiples facettes. Soulignons d'abord que, dans ses interventions publiques, le député cherche souvent à se définir comme le représentant d'une circonscription ou d'une région, faisant alors valoir les besoins de son milieu dans les différents secteurs d'intervention de l'Étar: éducation, santé, voirie, logement, loisirs, etc. «Ce rôle d'animateur régional, comme le soulignait Gaston Deschênes, met le député en relation avec des groupes représentant des communautés locales (...) qui requièrent ses services pour transmettre leurs idées, demander ou fournir des informations, obtenir de l'aide ou des services. " ${ }^{8} \grave{A}$ ceci s'ajoutent de multiples rencontres avec ses électeurs soit pour régler des problèmes d'ordre personnel en relation surtout avec l'appareil administratif, soit pour leur obtenir certains biens ou

7. Harold D. Clarke, Richard G. Price et Robert Krause, "Constituency Service among Canadian Provincial Legislators", Canadian Journal of Political Science / Revue canadienne de science politique, VIII (4), décembre 1975, 520-542.

8. Gaston Deschênes, op. cit., 34. 
services, soit même à titre d'agence de renseignements auprès de la population.

Ce rôle d'intermédiaire s'est profondément transformé depuis un siècle. À une époque où les moyens de communication étaient peu développés, les députés servaient encore largement de canaux et de moyens d'expression des désirs et besoins de la population. Le Parlement pouvait alors être considéré comme un véritable forum national où il appartenait à ses membres de dégager des solutions appropriées aux problèmes de la société en se basant sur la connaissance de certains faits et sur leurs relations avec les électeurs.

De nos jours, avec la croissance des interventions de l'État, les autorités politiques doivent le plus souvent s'assurer du concours des forces économiques et sociales qu'elles cherchent à contrôler et à réglementer de sorte qu'elles doivent se montrer sensibles à la pression et aux revendications de ces forces. Les groupes de pression ont alors tendance à devenir les interlocuteurs les plus privilégiés des gouvernements sans passer par le circuit des parlementaires qui perdent ainsi leur importance comme courroies de transmission entre les deux. Par contre, face à l'interventionnisme étatique et devant la croissance de l'appareil administratif qui en résulte, le député va servir de plus en plus d'intermédiaire entre la population et cette lourde machine administrative.

Avec le développement des moyens de communication modernes, le gouvernement non seulement transige directement avec les groupes, mais s'adresse aussi directement à la nation sans être obligé de recourir constamment aux députés comme propagandistes des politiques ministérielles. Le député sert de moins en moins "d'intermédiaire naturel» entre ses électeurs et le gouvernement au profit des groupes et surtout des médias qui en viennent à remplacer le Parlement comme forum national. ${ }^{9}$ 49-51.

9. Sur les transformations évoquées ici, voir Réjean Pelletier, article cité, surtout 
Le triple rôle de législateur, contrôleur et intermédiaire qu'est censé remplir le député québécois doit donc être évalué dans une juste perspective si l'on veut bien saisir le sens des différentes réformes parlementaires amorcées au Québec depuis une vingtaine d'années. Dans un Parlement qui ne légifere plus vraiment, n'ayant plus la maîtrise des projets de lois qui y sont présentés, dans un Parlement davantage contrôlé par l'exécutif que contrôleur de ce même exécutif et de son administration, dans un Parlement qui n'est plus réellement le grand forum de la vie politique nationale puisque d'autres l'ont remplacé à cet égard, il fallait s'attendre à ce que le «simple député» se sente souvent mal à l'aise dans son rôle de législateur, de contrôleur et même d'intermédiaire. Il fallait s'attendre également à certaines récriminations de sa part en vue d'améliorer sa situation.

\section{Le député - législateur}

À la suite de plaintes de certains députés souhaitant participer davantage aux travaux législatifs, le premier ministre Lesage confiait en 1963 à Jean-Charles Bonenfant la tâche de soumettre un document sur la réforme parlementaire. Cette étape marquait le début d'un long processus visant à modifier nos institutions parlementaires et surtout à en améliorer le fonctionnement.

Il semble que l'on ait fixé, dès cette époque, les balises des futures réformes quant au rôle du député - législateur: d'une part, on ne croit pas beaucoup au droit du député de présenter des lois en son propre nom et, en corollaire, on ne cherche pas vraiment à lui donner tous les moyens nécessaires pour exercer véritablement ce droit d'initiative en matière de législation; d'autre part, on souhaite le faire participer plus activement aux travaux législatifs, par le biais de commentaires, suggestions et critiques, en créant des groupes plus restreints où le député pourrait se faire entendre. Déjà la voie était tracée pour les futures réformes. En 
juillet 1964, on créait deux nouveaux comités permanents, celui de l'Éducation et celui des Régies gouvernementales, en plus de suggérer la création d'un comité spécial chargé d'examiner les crédits d'un ou de deux ministères (une entente à cet effet n'interviendra qu'en mars 1965).

Il ne s'agissait donc pas de dépouiller le gouvernement de son pouvoir législatif réel; tout au plus cherchait-on à redonner au député une infime partie de ce pouvoir en le faisant participer plus directement au processus législatif. Le moyen tout désigné pour assurer cette réforme, celui qu'on a pratiquement toujours considéré comme la panacée la plus susceptible d'améliorer la situation du député: le travail en commissions parlementaires. C'est ainsi que la réforme de 1969 créait 25 commissions parlementaires. Leur nombre fut réduit à 16 en mars 1973, pour remonter à 27 en 1978 et redescendre à 9 seulement en mars 1984. Créées le plus souvent en fonction des ministères existant, ces commissions étaient habituellement chargées d'étudier les crédits, les projets de lois et toute autre question relevant du (ou des) ministère(s) concerné(s).

Dans ce jeu de yo-yo, on perçoit vite toute l'ambiguité de cette première grande réforme: on se soucie certes de revaloriser le rôle du simple député en inventant des moyens pour le faire participer davantage aux travaux législatifs, mais il ne s'agit pas de rompre avec la forme d'évolution de notre système parlementaire, caractérisée par le passage de la souveraineté législative des mains du monarque à celles du gouvernement. Tout se passe comme si on voulait surtout occuper le député - tout en accélérant le travail parlementaire - sans en faire un véritable législateur, rôle qui demeurera toujours l'apanage du gouvernement. Le député qui est membre d'une commission parlementaire se borne plutôt à bonifier, amender ou critiquer un projet de loi dont le principe a déjà été adopté à l'Assemblée nationale. Sa marge d'intervention se trouve ainsi réduite à amender un projet sans altérer fonda- 
mentalement son contenu, contrairement à la situation qui prévaut dans les commissions américaines qui ont toute latitude à cet égard.

On ne considère pas encore le député comme un initiateur en matière de législation, mais plutôt comme le récepteur d'un projet dont le contenu initial est fixé au Conseil des ministres. Certes, on souhaite vivement l'impliquer plus directement dans le processus législatif, mais à la condition qu'il se cantonne à la discussion, à la critique et surtout à l'approbation de la législation, sans lui donner la capacité réelle de participer à la définition du contenu ou à l'élaboration des politiques.

Ce que les députés réclament depuis longtemps - et plus particulièrement les députés ministériels - c'est d'être associés plus étroitement à la formulation des politiques. Déjà, les plaintes des députés au début des années 60 avaient permis d'enclencher le processus de la réforme parlementaire. Lors d'un long débat sur ce même sujet en février 1967, le député Yves Michaud réclamait également la participation des députés à l'élaboration des lois. Avec l'arrivée de 102 députés ministériels à l'automne 1973, on pouvait s'attendre à ce que la «grogne» et le mécontentement s'emparent assez rapidement de tous ces députés non promus au conseil des ministres ou à des postes d'adjoints parlementaires. Comme le soulignait l'ex-ministre Raymond Garneau, "un des problèmes que nous avons, comme députés, c'est que lorsqu'on se présente devant l'électorat, on se présente avec l'intention d'être membre du gouvernement et on s'aperçoit, à un moment donné, qu'on est élu député. C'est de là que vient une bonne partie de la frustration. ${ }^{10}$ C'est précisément pour faire taire ces frustrations que le vice-président de l'Assemblée nationale, Me Robert Lamontagne, présentait en juin 1975 un rapport pro-

10. Dans Le parlementarisme britannique : anachronisme ou réalité moderne?, Québec, Assemblée nationale du Québec, coll. Documents et débats, 1980, 76. 
posant «d'intégrer davantage le travail du député ministériel à celui de ses collègues au Conseil des ministres».

Dans ce même esprit, le comité présidé par le député Gilles Michaud ${ }^{11}$ soumettait un rapport d'étape en juin 1979 dont l'un des axes de réforme reposait sur l'accroissement de l'indépendance du pouvoir législatif face au pouvoir exécutif. Selon ce comité, il fallait accorder une autonomie accrue aux commissions parlementaires, en particulier à leurs présidents, comme moyen de réaffirmer la souveraineté du Parlement.

C'est probablement le député Denis Vaugeois qui a poussé le plus loin cette réflexion sur le pouvoir législatif de l'Assemblée nationale et de ses membres ${ }^{12}$. Reconnaissant que le gouvernement avait accédé au rôle de législateur, il ajoutait aussitôt que "le Parlement n'en demeure pas moins la seule source d'information qui permette aux citoyens d'évaluer la qualité de leurs représentants. " ${ }^{13}$ C'est pourquoi il recommandait d'en faire un véritable forum démocratique", un lieu où soit favorisé le débat public sur la législation.

Cette recommandation se situe dans la lignée de certaines revendications antérieures. Mais il allait beaucoup plus loin que celles-ci en réclamant que l'Assemblée nationale puisse former des commissions d'initiatives parlementaires par opposition aux commissions d'initiatives gouvernementales. Un tel rôle d'initiative attribué aux parlementaires, ajoutait-il, permettrait de contrebalancer quelque peu cette influence grandissante de la fonction publique sur les politiques gouvernementales ${ }^{14}$. Son souci n'était

11. Comité sur la réforme parlementaire créé à la demande du ministre Robert Burns et composé du député Gilles Michaud à titre de président et des députés Roland Dussault, François Gendron, Richard Guay et Adrien Ouellette comme autres membres.

12. Denis Vaugeois, L'Assemblée nationale en devenir. Pour un meilleur équilibre de nos institutions, Québec, 28 janvier 1982, 202 p.

13. Ibid., 117.

14. Ibid., 128-129. 
certes pas de rendre aux parlementaires leur fonction première de législateur au sens strict, ce qui lui paraissait utopique, mais plutôt d'assurer un meilleur équilibre entre le gouvernement, la fonction publique et l'Assemblée nationale.

Comme on peut le constater, les suggestions n'ont pas manqué depuis une vingtaine d'années en vue d'améliorer le rôle du député comme législateur. Toutes se sont butées sur cette réalité fondamentale de notre système parlementaire: à savoir que le gouvernement ou l'exécutif se confond pratiquement avec le législatif: il est devenu le seul véritable législateur. Le travail en commission parlementaire n'a pas vraiment redonné aux députés leur rôle de législateur, d'autant plus que les commissions ont toujours reçu leurs mandats du gouvernement. Ne disposant pas d'une réelle autonomie, elles sont apparues comme "des outils commodes aux mains de l'exécutif ». ${ }^{15}$

Mais une tendance nouvelle semble se dessiner depuis l'adoption d'un nouveau règlement en mars 1984. Refusant la formule des commissions fonctionnelles préconisée dans le rapport Vaugeois, les parlementaires ont préféré maintenir la formule des commissions sectorielles et multifonctionnelles dotées cependant d'une plus grande autonomie et d'un certain pouvoir d'initiative afin qu'elles puissent remplir pleinement leurs mandats. Ainsi, les commissions élisent désormais un président et vice-président parmi leurs membres. Elles peuvent également étudier, de leur propre initiative, les projets de règlement et les règlements, les orientations, les activités et la gestion des organismes publics, les engagements financiers ou toute autre matière d'intérêt public. ${ }^{16}$

Une telle orientation semble à la fois réaliste et réalisable. Il apparaît utopique de vouloir faire des députés les seuls législateurs;

15. Gaston Deschênes, "Les réformes parlementaires ou le mythe de Sisyphe", à paraitre dans Le contrôle de l'administration et la réforme parlementaire, ENAP, 1984.

16. Article 117 des Règles de procédure et autres dispositions pertinentes, Assemblée nationale, 1984. 
le gouvernement va demeurer, qu'on le veuille ou non, le principal législateur dans notre système parlementaire. Mais il est possible de briser son monopole en redonnant aux députés un certain pouvoir d'initiative législative. C'est là une façon d'assurer un meilleur équilibre entre l'Assemblée nationale et le gouvernement.

\section{Le député - contrôleur}

Au moment où l'on se penchait sur le sort du député en tentant de le faire participer davantage aux travaux législatifs, on cherchait également à améliorer la situation du gouvernement en accroissant son efficacité. Cette double orientation montre toute l'ambiguïté et l'ambivalence des réformes entreprises depuis vingt ans. Un bon nombre d'entre elles, surtout dans les années 60 et au début des années 70 visait à accélérer les travaux en Chambre afin de rendre le Parlement (faudrait-il dire le gouvernement?) plus efficace. Dans la présentation du nouveau règlement adopté en 1972, le président Jean-Noël Lavoie écrivait: «Nous avons voulu assujettir le règlement à la réalité de l'Assemblée pour qu'il fasse de celle-ci un parlement moderne, efficace, qui permetrra à la majorité de faire adopter sa législation, à l'opposition de s'exprimer en toute liberté, à l'opinion publique de se manifester et à tous les députés de remplir pleinement leur rôle de législateur».

Le moyen privilégié pour accroître l'efficacité gouvernementale fut de restreindre le travail en Chambre, où théoriquement tous les ministres doivent être présents et où les débats sont habituellement longs, au profit d'un travail en commission parlementaire où ne siège ordinairement qu'un seul ministre. Dans cette foulée, on a cherché à gagner du temps en réduisant la durée de la période des questions, du débat sur le discours inaugural, et du débat sur le discours du budget, réduisant par le fait même le nombre des intervenants, tout en améliorant la procédure de vote. Ceci permettait de déplacer une partie des activités de la Chambre vers 
les commissions parlementaires, restreingnant l'exercice du pouvoir de contrôle en Chambre au profit d'un contrôle accru en commission.

Une première réforme en ce sens s'est traduite par la création d'une commission des engagements financiers, puis par l'étude des crédits en commissions parlementaires, laissant ainsi de côté le traditionnel comité plénier des subsides. En novembre 1977, on mettait à l'essai un règlement sessionnel comprenant, entre autres, l'abolition de la séance du vendredi et son remplacement par «la question avec débat». La réforme de 1984 vient d'abolir la question avec débat pour la remplacer par la procédure d'interpellation. Dans tous ces cas, c'est avant tout au bénéfice des députés de l'opposition qu'on a effectué ces réformes plutôt qu'à celui des députés ministériels. C'est le cas tout particulièrement de la procédure d'interpellation réservée aux seuls députés de l'opposition. ${ }^{17}$ On retrouve ici la distinction précédemment établie par André Gélinas entre oppositionnistes contrôleurs et ministériels propagandistes. L'exercice plus efficace de la fonction de contrôle profite avant tout au député de l'opposition et non au député ministériel.

Par un curieux paradoxe, au moment où l'on tente d'améliorer les rôles de législateur et de contrôleur du député face à un gouvernement déjà considéré comme tout-puissant, on cherche à assumer une plus grande efficacité au travail du gouvernement en accélérant les travaux en Chambre. On a ainsi davantage permis à la majorité ou au gouvernement de faire adopter sa législation qu'à l'opposition de s'exprimer en toute liberté. Certes, les différentes réformes n'ont jamais cherché à brimer la liberté d'expression des membres de l'opposition, mais plutôt à réduire le nombre des intervenants et la durée des interventions et des débats. On n'enlève

17. L'article 287 du Règlement de 1984 se lit comme suit: "Tout député d'opposition peut interpeller un ministre sur toute question d'intérêt général relevant de sa compétence." 
pas le droit de parole, mais on en réduit le temps d'utilisation dans un lieu qui est précisément consacré à la délibération et qui se veut un forum public.

Même le rapport d'étape du comité présidé par le député Gilles Michaud se proposait d'améliorer l'efficacité de l'appareil législatif et en faisait l'un de ses cinq grands axes de réforme. Encore une fois, le rapport Vaugeois se démarque assez nettement de cette orientation en tablant davantage sur l'exercice du contrôle que sur l'efficacité du Parlement. Trois séries de recommandations tendent vers cet objectif. D'abord, il s'agit de reconnaître à l'Assemblée nationale la responsabilité du contrôle de l'administration et des entreprises publiques en lui donnant «le pouvoir de «questionner» directement l'administration publique, d'identifier les faiblesses de gestion et de proposer des mesures pouvant améliorer l'efficacité administrative " ${ }^{18}$. Face aux administrateurs interrogés, le député ministériel pourrait se retrouver sur le même pied que son collègue de l'opposition.

Il faudrait également, selon le rapport Vaugeois, permettre à l'Assemblée nationale une meilleure étude du budget et une surveillance plus poussée des dépenses publiques. ${ }^{19}$ Rappelant que les grandes batailles de nos parlementaires avaient principalement pour objectif de leur donner le contrôle des dépenses publiques, le député Vaugeois constatait en même temps que le rôle des députés dans l'étude et l'approbation du budget avait été passablement réduit depuis ce temps. Sans remettre en cause le rôle prédominant de l'exécutif en matières budgétaires, il proposait plutôt d'axer le débat public non pas tant sur les contenus et les aspects de détail que sur les retombées et les effets d'entraînement, replaçant toutes les discussions sur le budget dans leur cadre véritable incluant, entre autres, les accords fiscaux avec le gou-

18. Rapport Vaugeois déjà cité, 91 .

19. Ibid., 99. 
vernement fédéral, la politique salariale du gouvernement et la politique d'aménagement du territoire.

Troisième volet de cette fonction de contrôle: confier à l'Assemblée nationale l'évaluation de la règlementation. Il s'agit d'abord et avant tout «de s'assurer que l'autorité administrative chargée de la législation subordonnée a bien rempli son mandat et qu'elle n'a excédé ses pouvoirs d'aucune façon. ${ }^{20}$. Ce thème figurait déjà dans les recommandations du rapport Bonenfant en 1964. Il fut repris dans plusieurs études depuis une dizaine d'années: d'une sous-commission créée en 1975, au comité Michaud en 1979, au projet de loi de l'ex-ministre Claude Forget en 1979, au rapport Vaugeois en 1982 jusqu'à la Commission d'étude sur le contrôle parlementaire de la législation déléguée qui remit son rapport en juillet $1983 .^{21}$

Il reste donc beaucoup à faire, en particulier sur le contrôle de l'administration et des organismes publics et sur le contrôle de la législation déléguée. Mais on a tout de même cherché à améliorer certains moyens de contrôle entre les mains des parlementaires, surtout ceux de l'opposition. C'était là une façon de reconnaître que, dans notre système parlementaire, s'exerce une véritable domination du gouvernement et du parti ministériel qui ne peut être tempérée que par la critique et le contrôle émanant des partis d'opposition (et de l'extérieur du Parlement, il va sans dire). L'accroissement de l'efficacité gouvernementale, estimait-on, ne devait pas se faire au détriment d'un contrôle accru des activités du gouvernement et de l'administration. Les différentes réformes entreprises au Québec n'ont pas remis fondamentalement en cause cette nécessaire dialectique entre le gouvernement et l'opposition. Mais on a oublié trop souvent les autres

20. Ibid., 110

21. Voir le rapport intitulé Le contrôle parlementaire de la législation déléguée, juillet 1983, 159 pages. La Commission était présidée par le député Denis Vaugeois, le député Richard French agissant à titre de vice-président. 
membres du parti ministériel qui ne sont là que pour servir de troupe docile et d'appui majoritaire au gouvernement en place.

\section{Le député - intermédiaire}

Si les députés des années soixante voulaient participer plus activement aux travaux parlementaires, ils ont également revendiqué, en corollaire, d'être dotés de moyens d'action leur permettant d'assumer pleinement leurs différents rôles. Ce sont surtout les moyens d'action reliés à leur rôle d'intermédiaire qui ont été les plus développés. On pouvait facilement aboutir à une entente entre tous les partis puisque ces moyens profitaient également aux députés des deux côtés de la Chambre.

Comme le travail du député était progressivement devenu un travail permanent, il fallait d'abord songer à lui accorder des indemnités plus généreuses.. L'année 1965 a été marquante à cet égard puisqu'auparavant la loi prévoyait que le député recevait une indemnité quotidienne si la session durait moins de trente jours et une indemnité sessionnelle lorsqu'on dépassait trente jours. Depuis cette date, la rémunération du député a été établie sur une base annuelle et augmentée régulièrement. À cette indemnité de base s'ajoutent désormais d'autres indemnités reliées à des fonctions gouvernementales (ministres) ou parlementaires (président, vice-présidents, adjoints parlementaires, présidents de commission, etc.).

L'Assemblée a accepté, au fil des ans, de fournir au député diverses allocations pour entretenir un bureau dans sa circonscription, défrayer les coûts de son personnel de bureau ainsi que pour assurer la location d'une résidence secondaire à Québec. Elle a accepté également de lui assurer certains frais de déplacement et de lui rembourser des frais de communication. Depuis février 1984, le député dispose d'une enveloppe globale pour embaucher son per- 
sonnel à la condition qu'au moins le tiers de cette somme soit dépensé à Québec et un autre tiers dans sa circonscription.

Tous ces moyens - ainsi que d'autres comme l'abolition de la séance du vendredi - tendent à favoriser une présence accrue du député dans sa circonscription, même s'il est appelé à passer de plus en plus de temps dans la capitale. Ils lui permettent par la même occasion de mieux partager son temps entre les travaux de la Chambre et le travail dans sa circonscription afin de remplir plus adéquatement ses multiples rôles.

Cependant, le député est souvent coïncé dans son rôle d'intermédiaire, entre les multiples demandes de ses électeurs et les démarches effectives qu'il peut accomplir auprès de la fonction publique qui se prétend neutre et craint constamment les pressions de nature trop partisane de la part des élus du peuple. Chargé de représenter et de défendre les intérêts de ses électeurs auprès du gouvernement et de l'administration, le député est certainement bien placé pour sensibiliser la fonction publique à la dimension politique des problèmes par delà leur dimension technique ou bureaucratique. Il ne s'agit certes pas de rendre la fonction publique partisane, mais plutôt de la "politiser» afin qu'elle n'oublie pas cet aspect des problèmes lorsqu'il s'agit de proposer des solutions. Bien que concurrencé par les groupes et les médias, le député se trouve encore au centre même de la vie politique nationale et, à ce titre, il devrait être l'un des interlocuteurs les plus privilégiés du gouvernement et de l'administration.

\section{Une orientation nouvelle}

Par rapport aux réformes réalisées ces dernières années, une tendance nouvelle semble se dessiner avec la réforme proposée récemment par le président Richard Guay. ${ }^{22}$ Celle-ci constitue

22. On peut consulter à ce sujet le Projet de réforme parlementaire du président Richard Guay en date du 22 juin 1983 et le nouveau règlement de l'Assemblée nationale paru en mars 1984 sous le titre Règles de procédure et autres dispositions pertinentes. 
l'aboutissement du travail fait antérieurement par divers comités. Dans son rapport d'étape, le comité Michaud définissait les deux axes premiers de toute réforme parlementaire en termes de «réaffirmation de la souveraineté du Parlement en tant que pouvoir délégué par le peuple» et d'accroissement «de l'indépendance du pouvoir législatif face au pouvoir exécutif».

De même, le rapport Vaugeois se fixait comme premier objectif de «redonner à l'Assemblée nationale un minimum d'indépendance» dans le sens où le Parlement pourrait se doter d'une administration autonome, distincte de celle du gouvernement, capable de gérer librement son budget et de se structurer de façon à favoriser l'initiative parlementaire. Sur cet objectif de base viendraient se greffer d'autres grandes priorités qui visent toutes à rééquilibrer le rapport de forces entre le Parlement, le gouvernement et l'administration.

Selon cette optique, on cherche moins désormais à améliorer la situation du député comme législateur, contrôleur ou intermédiaire, ou à accroître l'efficacité des activités gouvernementales qu'à assurer une plus grande autonomie à l'Assemblée nationale. À la traditionnelle fusion des pouvoirs législatif et exécutif, on veut substituer une plus grande indépendance de la branche législative en tentant de briser la soumission séculaire du législatif à l'égard de l'exécurif. Cetre orientation nouvelle est fondamentale et permettrait d'assurer un meilleur équilibre entre le Parlement et le gouvernement.

Deux réformes récentes traduisent bien cette orientation. D'abord, la création du Bureau de l'Assemblée nationale dirigé par le président de la Chambre et composé de sept autres députés (les ministres étant exclus) dont quatre du parti ministériel et trois représentants de l'opposition. En plus de donner son accord à toute réorganisation administrative de l'Assemblée, le Bureau est chargé d'en approuver le budget. C'est là une disposition essentielle permettant d'assurer l'indépendance tant recherchée 
face à la tutelle du Conseil du trésor. En plus de garantir la traditionnelle indépendance du député dans l'exercice de ses fonctions en lui accordant un certain nombre de droits, privilèges et immunités, la loi de l'Assemblée nationale établit désormais l'indépendance de la Chambre quant à son administration et à son budget.

La seconde réforme vise à assumer une plus grande autonomie des commissions parlementaires comme émanations du pouvoir législatif. En vertu du nouveau règlement sessionnel adopté en 1984, les présidents et vice-présidents des commissions sont élus par et parmi les membres de leurs commissions respectives de façon à assumer une meilleure autonomie face au gouvernement. Les présidents sont également appelés «à jouer un rôle primordial dans l'organisation et la planification des travaux". En outre, les commissions déterminent elles-mêmes l'organisation et le déroulement de leurs travaux, bien que cette autonomie accrue ne doive pas altérer "l'initiative du leader parlementaire du gouvernement à l'égard de la conduite des travaux de la Chambre". À des mandats confiés par l'Assemblée (à toutes fins utiles, par le leader du gouvernement) s'ajoutent désormais des mandats dont se saisissent elles-mêmes les commissions. On cherche en somme à mieux baliser le travail du leader du gouvernement et à se rapprocher de l'autonomie des commissions américaines, mais sans en arriver à une dissociation complèt de l'exécutif et du législatif.

\section{Conclusion}

Les réformes récentes, tout en traduisant une orientation nouvelle, ne tranchent pas radicalement avec les situations antérieures. Même si elles veulent assurer une plus grande indépendance de l'institution parlementaire face à la domination beaucoup trop forte et trop exclusive du gouvernement, ces réformes sont encore placées «sous le signe de la valorisation du rôle par- 
lementaire du député et d'une plus grande efficacité des travaux de l'Assemblée et de ses commissions. " ${ }^{23}$

On s'est beaucoup soucié jusqu'ici de revaloriser le rôle du député comme législateur et comme contrôleur et d'améliorer sa situation matérielle. Il était normal d'en faire le pivot de toute réforme puisqu'il est l'élément de base de l'Assemblée nationale. Aujourd'hui, on tente d'aller plus loin en favorisant une plus grande autonomie de la Chambre elle-même face à la tutelle gouvernementale. Pour ma part, i'y vois une orientation positive qui doit être scrutée encore davantage.

Mais les différentes réformes parlementaires jusqu'à ce jour ont reproduit le plus souvent l'image de Janus. Marquées du sceau de l'ambiguité, elles ont voulu faire du député le pivot des réformes, revaloriser son rôle, assurer une plus grande indépendance au Parlement, tout en veillant en même temps à ce que le gouvernement puisse faire adopter son programme législatif sans trop de contraintes. La conclusion du président Richard Guay dans son projet de réforme parlementaire traduit cette double orientation et cette constante ambivalence: assurer à chaque député que l'organisation et le fonctionnement du Parlement lui permettront toujours d'y jouer son rôle librement et de s'y sentir utile et en même temps permettre au gouvernement, en lui laissant toute l'initiative nécessaire, de réaliser le programme qu'il s'est tracé dans le message inaugural.

Comment réaliser un habile dosage entre les deux? C'est là la clé des réformes futures qui devraient justement permettre d'en arriver à un meilleur équilibre entre le Parlement, le gouvernement et l'administration. Mais encore faudrait-il que le Parlement consente à se réformer ... et à s'affirmer davantage, sans toujours attendre l'impulsion ou l'accord du gouvernement.

23. Projet de réforme parlementaire du président Richard Guay, juin 1983, 20. 